\title{
EXOGAM at the ILL: the EXILL campaign
}

\author{
Gilles de France ${ }^{1, *}$, Aurélien Blanc ${ }^{2}$, Michael Jentschel ${ }^{2}$, Ulli $\mathrm{Köster}^{2}$, Silvia Leoni ${ }^{3}$, Paolo \\ Mutti $^{4}$, Gary Simpson ${ }^{2}$, Torsten Soldner ${ }^{2}$, and Waldemar Urban ${ }^{5}$ \\ ${ }^{1}$ GANIL, CEA/DRF-CNRS/IN2P3, BP 55027, 14076 Caen cedex 5, France \\ ${ }^{2}$ ILL, Institut Laue-Langevin, BP 156, 6, rue Jules Horowitz, 38042 Grenoble Cedex 9, France \\ ${ }^{3}$ University of Milano and INFN sez. Milano,Via celoria 16, 20133 Milano, Italy \\ ${ }^{4}$ LPSC, Université Grenoble Alpes, 38026 Grenoble Cedex, France \\ ${ }^{5}$ Faculty of Physics, University of Warsaw, ul. Hoża 69, 00-681 Warsaw, Poland
}

\begin{abstract}
Radiative capture reactions on rare targets and prompt spectroscopy of neutron-rich nuclei produced in the neutron-capture induced-fission of ${ }^{235} \mathrm{U}$ and ${ }^{241} \mathrm{Pu}$ have been performed at the Institut Laue-Langevin (ILL). The highefficiency germanium (Ge) EXOGAM array has been used, complemented with Ge detectors from GASP and the ILL. $\mathrm{LaBr}_{3}$ detectors from the FATIMA collaboration have also been coupled to EXOGAM to measure lifetimes of lowlying excited states. The measured characteristics indicate very good performances of the overall setup. Some recent results will be discussed.
\end{abstract}

\section{Introduction}

Fission produces neutron-rich nuclei in a very effective way. In particular, one of the best mechanism is to induce fission of actinide targets using cold neutrons from a neutron guide. In this regime, the incident neutrons bring just enough energy into the system to trig fission, whilst preserving the neutron-richness of the fragments. The average spin generated in this reaction is around 6-7 [1] and states with spins as high as $20 \hbar$ can be observed. Of particular interest is the use of ${ }^{235} \mathrm{U}$ and ${ }^{241} \mathrm{Pu}$ fissile targets which give access to nuclei that are too weakly produced using spontaneous fission sources or another production mean to perform their prompt spectroscopy. In addition to the spectroscopy of nuclei produced in such a cold fission process, neutron capture reactions using rare targets have also been performed. In this contribution we will describe the installation of the EXOGAM array, usually standing at GANIL, at the Institut Laue Langevin (ILL), Grenoble, and the experimental campaign which took place in 2012 and 2013.

\section{Physics case}

The basic idea of EXILL was to install a large number of efficient germanium detectors around a fissile target irradiated by a well collimated cold neutron beam. Large arrays like Euroball or Gammasphere were already used to study fission fragments produced in the spontaneous fission of ${ }^{252} \mathrm{Cf}$ and ${ }^{248} \mathrm{Cm}$ sources but it was the first time that such a large array was installed in a reactor facility, around a fissile target irradiated by cold neutrons. Traditional

\footnotetext{
*e-mail: defrance@ganil.fr
} 


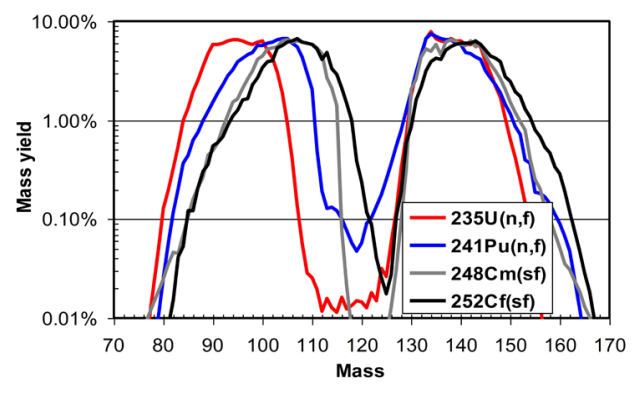

Figure 1. Mass yield distributions measured for the spontaneous fission of ${ }^{252} \mathrm{Cf}$ and ${ }^{248} \mathrm{Cm}$ compared to the thermal neutron induced fission of ${ }^{235} \mathrm{U}$ and ${ }^{241} \mathrm{Pu}$ (data from [2]).

nuclear physics at the ILL consists essentially in decay spectroscopy studies at the focal plane of the Lohengrin spectrometer. In the EXILL campaign instead we performed prompt spectroscopy. We used ${ }^{235} \mathrm{U}$ and ${ }^{241} \mathrm{Pu}$ targets giving access to many nuclei where little was known, especially in the regions north-east of ${ }^{78} \mathrm{Ni}$ and beyond ${ }^{132} \mathrm{Sn}$. This is what is shown in Figure 1 which compares the mass yield distribution from the thermal neutron induced fission of ${ }^{235} \mathrm{U}$ and ${ }^{241} \mathrm{Pu}$ and the yield from the spontaneous fission of ${ }^{252} \mathrm{Cf}$ and ${ }^{248} \mathrm{Cm}$. The production of nuclei in the mass A 90 region is an order of magnitude larger using a ${ }^{235} \mathrm{U}$ target compared to the two spontaneous fission sources. The same is true for nuclei in the mass A 125 region using a ${ }^{241} \mathrm{Pu}$ target. These two mass regions were the focus of the EXILL physics case.

In addition the high photopeak efficiency of the array allowed the study of samples that have low neutron capture cross sections of tens of millibarn or where only small milligram quantities of enriched material are available. An important property of capture reaction is its non selectivity which ensures the feeding of all available states in a certain energy and spin range for complete spectroscopy. Therefore, studies on collective models, symmetries and statistical properties of nuclei close to stability become possible.

The complete physics case of the EXILL campaign will not be detailed here but, to summarize it, we concentrated on a few topics which are:

- Shell Model (SM) nuclei with a few particles or holes outside a double shell closure

- Shapes and shape transitions

- Symmetry studies

- Statistical properties of nuclei

- Level densities

- Application to reactor physics

- Radioisotope for nuclear medicine

In this paper we will discuss a few examples illustrating the opportunities offered with the EXILL campaign.

\section{Setups}

Two setups have been operated (see reference [3] for a complete description). The first one consisted in 8 EXOGAM large clovers [4], 6 large coaxial detectors from GASP [5] and the 2 clovers from the ILL (see picture 2). In the second setup, the GASP and ILL detectors were replaced by $16 \mathrm{LaBr}_{3}$ crystals from the FATIMA collaboration [6].

The two setups were installed around the target point of the PF1B line of the ILL. The ILL reactor is the world's brightest continuous neutron source with an in-pile flux up to $1.5 \times 10^{15}$ 


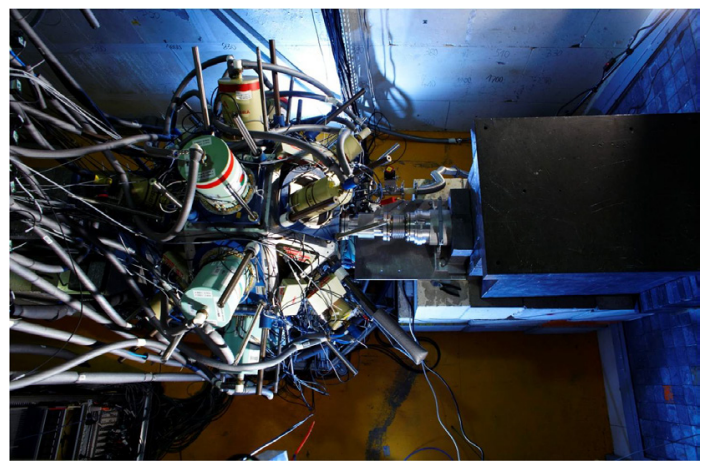

Figure 2. Picture of the spectroscopy setup of the EXILL campaign in the PF1B area. The neutron beam is coming from the right hand side.

neutrons per s.cm ${ }^{2}$. In order to produce around $10^{4}$ fissions per second in a $400 \mu \mathrm{g} / \mathrm{cm}^{2}$ thick, ${ }^{235} \mathrm{U}$ target a flux of $10^{8}$ neutrons per $\mathrm{s.cm}{ }^{2}$ is required. An efficient collimation system was developped which details can be found in [3]. The campaign covered 93 days of effective beam time for experiments.

In the absence of any fragment identification, it is crucial to acquire high-fold events (3 or larger) to possibly associate the observed $\gamma$-rays to a given fragment. This technique relies on the knowledge of the transitions deexciting the first excited states in the two fragments and the measurements of the evolution of the unknown $\gamma$-rays intensity gated by known transitions in a series of isotopes. ${ }^{235} \mathrm{U}$ and ${ }^{241} \mathrm{Pu}$ targets have been used leading for a given nucleus to two distinct complementary fragments, which greatly helps to confirm the assignment. The method relies therefore primarily on the efficient detection of high-fold data, which is achieved using a large number of efficient HPGe detectors. Such a technique is now well established and has been proven to work very well to analyze fission data $[8,9]$. The total photopeak efficiency for the $16 \mathrm{Ge}$ detectors setup was about $6 \%$ at $\mathrm{E}_{\gamma}=1.3 \mathrm{MeV}$. The second setup using the $\mathrm{LaBr}_{3}$ detectors was dedicated to lifetime measurements. In this configuration, the EXOGAM detectors are used to select the fragment of interest and the $\mathrm{LaBr}_{3}$ crystals give the lifetimes via the electronics fast timing technique using $\mathrm{LaBr}_{3}-\mathrm{LaBr}_{3}$ coincidences [10-13]. This method requires therefore $\mathrm{Ge}-\mathrm{LaBr}_{3}-\mathrm{LaBr}_{3}$ or $\mathrm{Ge}-\mathrm{Ge}-\mathrm{LaBr}_{3}-\mathrm{LaBr}_{3}$ coincidences. Recently, a novel method to analyze such data has been developed [14] and lead to the determination of lifetimes with an accuracy of 5 ps between $40 \mathrm{keV}$ and $6.7 \mathrm{MeV}$.

\section{Example of results}

\section{1 ${ }^{133} \mathrm{Sb}$ : Coupling collective and single-particle states in the vicinity of a doubly closed shell nucleus}

Shell Model nuclei like those located one or few particles away from ${ }^{132} \mathrm{Sn}$ are cornerstones of nuclear structure theories. In this context we have studied particle-core excitations in the one-valence proton nucleus ${ }^{133} \mathrm{Sb}$. For this we exploited the full complementarity of the various runs within the EXILL campaign i.e. spectroscopy and lifetime measurements using both ${ }^{235} \mathrm{U}$ and ${ }^{241} \mathrm{Pu}$ targets. In the spectroscopy part we looked for excited states above the $21 / 2^{+}$isomer. This was possible thanks to the capability of EXILL to build prompt-delayed and prompt-prompt coincidences (see Figure 3). Several prompt transitions were found in coincidence with known delayed transitions in ${ }^{133} \mathrm{Sb}$. Using the prompt-prompt coincidence relationships, we then could place these new transitions in the level scheme, above the isomer. We also used fission events from ${ }^{235} \mathrm{U}$ and ${ }^{241} \mathrm{Pu}$ targets to ensure that the newly observed transitions were observed in both cases hence not associated to a complementary fragment of ${ }^{133} \mathrm{Sb}$. 


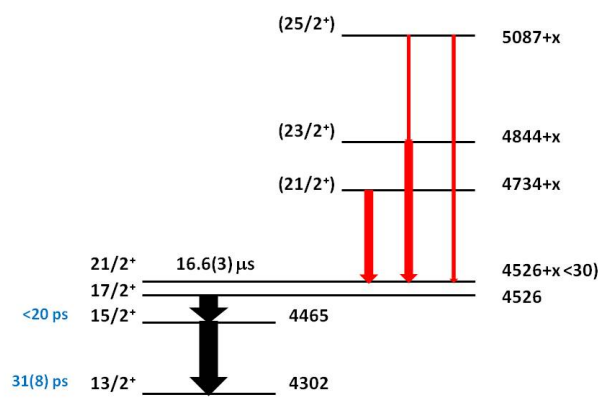

Figure 3. Partial level scheme of ${ }^{133} \mathrm{Sb}$ showing only states discussed in this paper with the newly observed $\gamma$-rays above the $21 / 2^{+}$isomer in red and the new lifetimes in blue.

Lifetime measurements were then used to investigate collective properties of the $15 / 2^{+}$ and $13 / 2^{+}$states located just below the isomer. These lifetimes were extracted using the centroid shift technique [12] from which the reduced transition probabilities of $B\left(M 1: 15 / 2^{+} \rightarrow\right.$ $\left.13 / 2^{+}\right)>0.24$ W.u and $B\left(M 1: 13 / 2^{+} \rightarrow 11 / 2^{+}\right)=0.0042(15)$ W.u were deduced. This difference of two orders of magnitude clearly reveals a profound modification in the structure of the two levels. In order to get insight into this puzzle a new model called the Hybrid Configuration Mixing Model was developed $[15,16]$ with the aim to couple collective and single-particle excitations in a fully self-consistent way. This model suggests that the low spin states are built from a $g_{7 / 2}$ protons coupled to the $2^{+}{ }^{132} \mathrm{Sn}$ core excitation while the higher lying levels arise from the valence proton coupled to single-neutron $h_{11 / 2}^{-1} f_{7 / 2}$ excitations. As a result the model gives $0.021 \mathrm{~W} . u$ and $0.001 \mathrm{~W} . u$ for the $15 / 2^{+} \rightarrow 13 / 2^{+}$and $13 / 2^{+} \rightarrow 11 / 2^{+}$transitions respectively, in fair agreement with data, which is not the case for shell model calculations [17].

\subsection{Symmetry studies}

Testing the validity of the $\mathrm{SO}(6)$ limit of the sd Interacting Boson Model $([18,19])$ is a long quest in symmetry studies. Using the fast timing part of the EXILL campaign, the Cologne group led by J Jolie proposed to study ${ }^{196} \mathrm{Pt}$ known to be a good candidate for such studies. In the $\mathrm{SO}(6)$ limit, the $\mathrm{E} 2$ transition operator is a generator of $\mathrm{SO}(6)$ and strictly speaking only transitions between states with $\Delta \sigma=0$ are allowed ( $\sigma$ being one of the quantum number associated to the irreducible representations of $\mathrm{SO}(6))$. Therefore, E2 transitions between different $\mathrm{SO}(6)$ representations $(\Delta \sigma \neq 0)$ are forbidden. The states of interest have low spin and high excitation energy and are well populated in $(n, \gamma)$ reactions. This was realized during the EXILL campaign using a ${ }^{195} \mathrm{Pt}$ target and using $\mathrm{Ge}-\mathrm{LaBr}_{3}-\mathrm{LaBr}_{3}$ coincidences to deduce the lifetimes of the third $0^{+}$state at $1402 \mathrm{keV}$ and the first $2^{+}$state at $356 \mathrm{keV}$ (which lifetime was already known at 49.2(2) ps). The measurements yielded the values of 50(5) ps for the $2_{1}^{+}$state and an upper limit of $12 \mathrm{ps}$ for the $0_{3}^{+}$state. Using also the lower limit from Börner et al [19], it was established that $0.56 \mathrm{~W} . \mathrm{u}<B\left(E 2 ; 0_{3}^{+} \rightarrow 2_{1}^{+}\right)<5$ W.u and $0.05 \mathrm{~W} . \mathrm{u}$ $<B\left(E 2 ; 0_{3}^{+} \rightarrow 2_{2}^{+}\right)<0.41$ W.u. The absence of collective $\mathrm{B}(\mathrm{E} 2)$ values demonstrates for the first time the validity of the $\mathrm{SO}(6)$ symmetry [20].

\section{3 ${ }^{195} \mathrm{Pt}$ : the link between nuclear structure and medical physics}

The structure of ${ }^{195} \mathrm{Pt}$ was also studied and more specifically the population of the $21 / 2^{+}$ isomer using the radiative capture ${ }^{194} \mathrm{Pt}(\mathrm{n}, \gamma)$ reaction. This nucleus has been recognized 
since long as a good candidate to probe multi-j supersymmetry ([21, 22]). In addition to this fundamental interest, ${ }^{195} \mathrm{Pt}$ is also a promising candidate for nuclear medicine since it combines therapy and diagnosis capabilities. Indeed its $T_{1 / 2}=4.01$ days isomer is compatible with such a usage (long enough for transportation and short enough for biology). This isomer decays via a highly converted M4 transition which provides an interesting possibility for treatment, with a very high linear energy transfer deposited within a few $\mathrm{nm}^{3}$, capable to induce double strand DNA breaks. In addition, the $99 \mathrm{keV}$ gamma-ray and 65-78 keV Xrays emitted in the cascade from the isomer allow imaging using Single Photon Emission Computed Tomography and gamma cameras. During the EXILL campaign we searched for new doorway states that would significantly feed the isomer and decay to the ground state. In this study several new states have been identified in the decay from the $1 / 2^{+}$neutron capturing state at $6.1 \mathrm{MeV}$. To envisage an abundant production of the isomer for nuclear medicine, a method to populate specifically the doorway state of interest has to be applied. For this, another experiment was realized at the HIGS facility (TUNL) where we tried to use a gamma beam at energies tuned around the newly identified states in order to measure the population of the isomer of interest. This work is still under analysis and is part of the thesis work of D. Wilmsen.

\section{Conclusion}

The EXILL campaign ran smoothly for two reactor cycles ( $\sim 100$ days) at the ILL, harvesting results from 73 proposals and gathering together 18 different laboratories. Today more than 30 papers have been published in refereed journals, showing the success of this adventure. The installation of a large and efficient gamma-ray array at a neutron beam facility is proven to be very effective in the study of neutron rich nuclei from cold neutron induced fission as well as in performing the detailed spectroscopy of close-to-stability nuclei in radiative capture reactions.

\section{Acknowledgements}

The EXILL campaign would not have been possible without the support of several services at the ILL and the LPSC. We are grateful to the EXOGAM collaboration for the loan of the detectors, to GANIL for assistance during installation and dismantling, and to the INFN Legnaro laboratory for the loan of the GASP detectors.

\section{References}

[1] M.A.C. Hotchkis et al., Phys. Rev. Lett. 64, 3123 (1990)

[2] JEFF-3.1.1 database, http://www.oecd-nea.org/dbdata/jeff/

[3] M. Jentschel et al., JINST 12 P11003 (2017)

[4] J. Simpson and the EXOGAM collaboration, Acta Physica Hungarica, New Series, Heavy Ion Physics 11159 (2000)

[5] D. Bazzacco and the GASP Collaboration, Proc. Int. Conf. Nuclear Structure at High Angular Momentum, Ottawa, AECL-10613 376 (1992)

[6] http://nuclear.fis.ucm.es/fasttiming/

[7] H. Abele et al., Nucl. Instr. and Meth. A 562407 (2006)

[8] M.A.C. Hotchkis et al., Nucl. Phys. A 530111 (1991)

[9] I. Ahmad and W.R. Phillips, Rep. Prog. Phys. 581415 (1995)

[10] K.E.G. Löbner The electromagnetic interaction in nuclear spectroscopy, (ed. W. D. Hamilton, North-Holland New York 1975) 173 
[11] H. Mach, R.L. Gill, M. Moszyński Nucl. Instr. and Meth. A 28049 (1989)

[12] J.M. Régis et al., Nucl. Instr. and Meth. A 62283 (2010)

[13] J.M. Régis et al., Nucl. Instr. and Meth. A 763210 (2014)

[14] J.M. Régis et al., Nucl. Instr. and Meth. A 68436 (2012)

[15] G. Bocchi et al., Phys. Lett. B 760, 273 (2016)

[16] G. Colò et al., Phys. Rev. C 95, 034303 (2017)

[17] W. Urban et al., Phys. Rev. C 62, 027301 (2000); Phys. Rev. C 79, 037304 (2009)

[18] J.A. Cizewski, R.F. Casten, G.J. Smith, M.L. Stelts, W.R. Krane, H.G. Börner, W.F. Davidson, Phys. Rev. Lett. 40167 (1978)

[19] H.G. Börner, J. Jolie, S.J. Robinson, R.F. Casten, J.A. Cizewski, Phys. Rev. C 42 R2271 (1990)

[20] J. Jolie et al., Nucl. Phys. A 9341 (2015)

[21] D.D. Warner, R.F. Casten, M.L. Stelts, H.G. Börner and G. Barreau, Phys. Rev. C 26 1921 (1982)

[22] R.F. Casten, G.G. Colvin and K. Schreckenbach, J. Phys. G 13221 (1987) 